\title{
The Role of Antimicrobial Peptides in Lung Cancer Therapy
}

Lucy Swithenbank and Claire Morgan*

Swansea University Medical School, Institute of Life Science, Swansea University, SA2, 8PP, United Kingdom

*Corresponding author: Claire Morgan, Swansea University Medical School, Institute of Life Science, Swansea University, SA2, 8PP, United Kingdom, Tel: 44 1792 606543; Fax: 441792 285201; E-mail: C.Morgan@swansea.ac.uk

Received date: January 28, 2017; Accepted date: February 3, 2017; Published date: February 10, 2017

Copyright: (c) 2017 Swithenbank L, et al. This is an open-access article distributed under the terms of the Creative Commons Attribution License, which permits unrestricted use, distribution, and reproduction in any medium, provided the original author and source are credited.

\begin{abstract}
Lung cancer is a major health burden accounting for 1.59 million deaths worldwide. For both sub-types of lung cancer (NSCLC and SCLC), chemotherapy is an option. However, success is limited and side effects are detrimental to an individuals' overall quality of life. To complicate matters further, cancer cells can develop resistance to chemotherapeutic agents due to the presence of membrane associated proteins such as P-glycoprotein and Multidrug resistance-associated protein. Thus it is, imperative that new drug treatments are developed that have neither the toxicity nor mechanisms of resistance associated with conventional chemotherapy.

In recent years, antimicrobial peptides (AMPs) have attracted attention as potential anti-cancer drugs due to reports that they can selectively target and kill cancer cells while leaving normal healthy cells unaffected. This review summaries several studies and discusses whether AMPs could be a viable treatment option in the fight against lung cancer.
\end{abstract}

Keywords: Antimicrobial peptides; Cancer; Lung cancer; Therapeutics; Anti-cancer

\section{Introduction}

In 2012 it was reported that there was an estimated 14 million new cancer cases and 8.2 million cancer related deaths worldwide with lung cancer being the most common cause of death [1]. In the UK and USA, lung cancer is the second most common cause of cancer for both males and females and accounts for around 35,400 and 158,100 deaths per year respectively [2,3]. The majority of lung cancers arise in epithelial cells and can broadly be divided into two sub-groups: nonsmall cell lung cancer (NSCLC) and small cell lung cancer (SCLC) which account for $85 \%$ and $15 \%$ of all lung cancer cases respectively. In addition there are three main sub-types of NSCLC; squamous cell lung carcinoma, large cell lung carcinoma and adenocarcinoma [4].

SCLC is treated mostly with chemotherapy whereas NSCLC can be treated with chemotherapy, surgery, radiotherapy or a combination of these treatments depending on the stage at which the cancer is diagnosed [2]. While several treatment options exist, chemotherapy remains the treatment choice for advanced/metastatic disease. Unlike other cancers such as breast and prostate, survival rates for lung cancer have not shown great improvements. Only $5 \%$ of people with lung cancer are alive 10 years after diagnosis as over two thirds are diagnosed at an advanced stage when curative treatment is not currently possible [2]. Thus, while chemotherapy is given to people with late stage lung cancer its success is limited. Furthermore, due to the fact chemotherapeutic drugs are non-specific in terms of the type of dividing cells they target they are also toxic to normal dividing cells causing deleterious side effects such as nausea, diarrhoea, hair loss, anaemia and infections. Toxic effects on normal cells aside, cancer cells can develop resistance to chemotherapeutic drugs due to the presence of membrane associated proteins such as P-glycoprotein and the multidrug resistance-associated protein (MRP) [5] that act as efflux pumps. With specific regard to lung cancer it has been reported that $90 \%$ of SCLC tumours will initially respond to chemotherapy, but patients almost always relapse with multidrug-resistant disease while the NSCLC tumours has a much lower response rate due to inherent drug resistance [6]. There is only a $10-20 \%, 5$ year survival rate for NSCLC due to the aggressiveness of the disease and the lack of effective treatments [7]. It is, therefore, imperative that new drug treatments are developed that have neither the toxicity nor mechanisms of resistance associated with conventional chemotherapy.

\section{Antimicrobial Peptides}

Antimicrobial peptides (AMPs) are a group of compounds that are a conserved element of the innate immune response [8]. Found in all species investigated such as bacteria, fungi, plants, insects, birds, fish, amphibians and mammals [8,9] these small peptides are between 12-50 amino acids long [10] and have been shown to effectively kill a wide range of micro-organisms such as viruses, fungi and both gramnegative and gram-positive bacteria [11]. AMPs in animals are expressed by a wide variety of cells such as epithelial cells of the stomach, skin, and respiratory tract, mast cells, monocytes and neutrophils [12]. To date, over 5000 AMPs have been discovered or synthesised [13]. AMPs are grouped into two sets; those which are toxic towards bacteria and cancer cells but not to mammalian cells and those which are cytotoxic towards bacteria, cancer cells and normal mammalian cells [14]. AMPs which are nontoxic towards mammalian cells hold great potential in cancer therapy. Not all AMPs possess anticancer properties but those that do, Anti-Cancer Peptides (ACPs), have been recognised to be more specific in their effects producing less harmful side effects than chemotherapy and traditional therapies [15]. Many different characteristics, properties and delivery combinations have been pinned as contributing factors for the ACPs to express their selective nature towards cancer cells. 


\section{Mechanisms Involved in AMP Induced Cell Death}

AMPs act by several mechanisms-barrel stave, toroidal, carpet model, and the aggregate channel model $[16,17]$. With the exception of the barrel-stave model, the other models are still open to disagreement regarding their mode of actions as well as currently lacking any clear distinction between their characteristics [17]. However it would appear that the overall mechanism favoured by the AMPs is dependent upon the amino acid sequence, amphipathicity, cationic charge and size of the peptide [18].

Barrel stave, the most widely accepted mechanism, is characterised by its process of membrane disruption, producing a highly organised cylindrical pore [19]. The AMP is attracted to the cancer cell (via the properties mentioned below). Once on the cell the AMP can bind to the cell membrane anchoring it into place [20]. The peptides then expose their hydrophobic amino acids to the lipid bilayer within the membrane, connecting the two membranes, forcing a conformational change in the membrane and forming a transmembrane pore (Figure 1) $[21,22]$. This pore formation results in the cellular material leaking out of the cell resulting in the cell death.

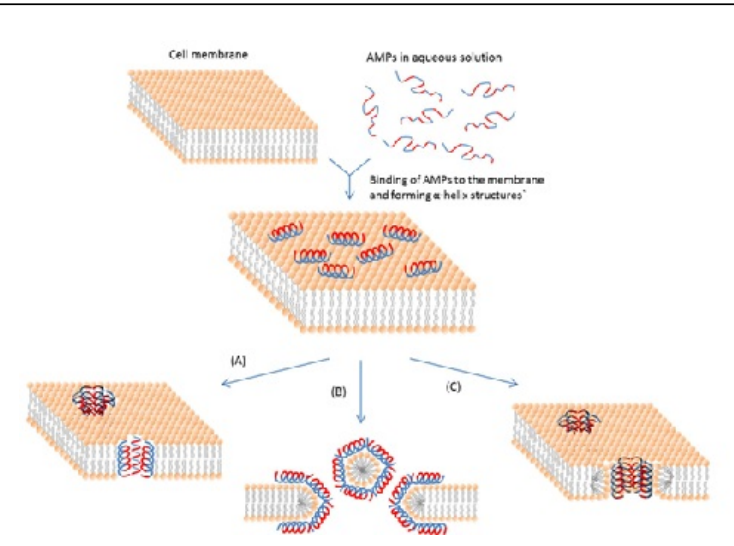

Figure 1: AMP induced mechanisms of death. The mechanisms of action by which AMPs act upon cell membranes resulting in cell death. (A) Barrel-Stave model. AMP molecules insert themselves into the membrane perpendicularly, connecting the two layers of the membrane to form a transmembrane pore. (B) Carpet model. Small areas of the membrane are coated with AMP molecules with hydrophobic sides facing inward leaving pores behind in the membrane. (C) Toroidal pore model. This model resembles integration between the AMP and the lipid bilayer [22].

The toroidal model is very similar in characteristics to those of the barrel stave model, in that they both form pores within the membrane of the target cell. However there are some important differences which distinguish the two models. In the toroidal model when the peptides expose the hydrophobic amino acid residues to the lipid core of the membrane- instead of forcing a conformational change-the peptides and lipids integrate together forming torus pores spanning the membrane [20]. The target cell membrane thins whilst the phosphate head groups of the lipids expand forcing the membrane bilayer to curve and bend [19]. Toroidal pores characteristically form very short

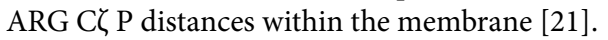

C $\zeta$ of ARG describes the distance between Arginine-Arginine residues within the membrane structure; the short distance is due to strong electrostatic interactions between the residues-typically shown close to the surface of membranes [23]. Once the channel is formed the intracellular material leaks out of the target cell leading to cell death. It has been suggested that the strongest bonding between AMPs and their target cell membrane is through the use of the toroidal method [24].

The carpet model (Carpet-like model) acts very differently to both the barrel stave and toroidal model. A key characteristic of this model highlighted by Liu et al. is the necessity for the AMP concentration to reach a threshold limit (AMP and cancer type dependent) in order for cell death to be instigated. Although a threshold is needed to instigate the mechanism, a high peptide to lipid ratio is also necessary [25]. The hydrophilic amino acids within the AMP sequences are attracted to the phospholipid heads of the membrane bilayer, as seen in Figure 2. The bound AMPs disrupt the curved anionic membrane, associating with the inner membrane of the target cell [26]. The peptides reorganise to surround the cell membrane and acts indirectly to dissolve the cell membrane, killing the target cell.

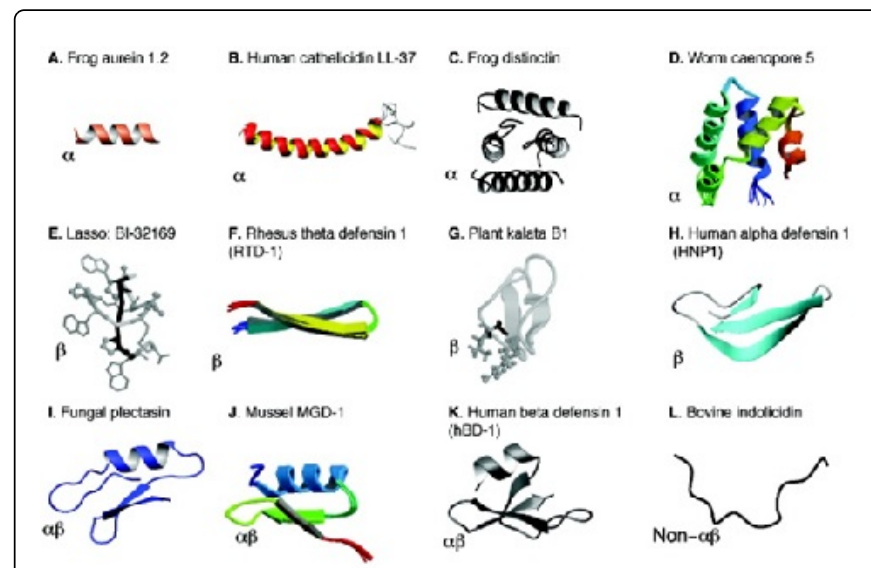

Figure 2: AMP structures. The structural diversity of naturally occurring AMPs. A-D; are $\alpha$ - helices, E-H; show the $\beta$ - sheet structure from differing perspectives, I-K; $\alpha \beta$ peptides, $L$; non- $\alpha \beta$.

\section{Properties of AMPs}

AMPs are specifically selective for cancer cells over normal epithelial cells and this is generally attributed to several complimenting characteristics between the cancer cell membranes and the AMPs. Such characteristics include their ability to interact electrostatically with each other, membrane fluidity, and cholesterol content of the cell membrane and the hydrophobicity of the AMP.

\section{Hydrophobicity and electrostatic interaction}

AMPs are generally cationic, that is they have a net positive charge of +2 to +7 due to an excess of basic amino acid such as arginine, lysine and histidine while $50 \%$ or more of the amino acids are hydrophobic [27]. In contrast to the positive charge of the AMPs, cancer cells typically have a net negative charge due to over expression of anionic molecules and increased o-glycosylated mucins on their surface [28]. The contrasting net charges induce an electrostatic interaction between the AMPs and the cancer cell membrane. The electrostatic interaction creates a strong binding of the AMP onto the target cell and provides specificity for the cancer cells over normal epithelial cells. 
While hydrophobicity has been traditionally associated with the effectiveness of AMPs to act as anti-cancer agents [20,29] this theory has now been adapted to recognise that hydrophobicity and effectiveness (represented as therapeutic index) are not directly correlated as was once previously thought. Several studies have been carried out testing the relationship between hydrophobicity and therapeutic index concluding that the relationship is a bell curve rather than a linear correlation [25,30,31]. As hydrophobicity of the AMPs increase the potency and therapeutic index also increases until a threshold is reached-dependent upon the AMP and the cancer type. As hydrophobicity increases past this point it has a detrimental impact upon therapeutic index [25,30]. Huang et al. [25] investigated this relationship in the lung cancer cell line A549. They showed that a middle range of hydrophobicity- $\sim 10-35 \mu \mathrm{mol} / \mathrm{l}$, therapeutic index $=15.8$ to 24.6 -resulted in a three/fourfold increase in the therapeutic index compared to the low or high hydrophobic levels, therapeutic index $=8.8$ to 0.5 and 9.1 to 4.0 respectively. The reduction of therapeutic index results as the increase in hydrophobicity and potency compromises the selectivity of the AMPs for the target cells [30]. With reduced selectivity the AMPs are unable to bind to the cancer cells as specifically reducing the chances of cell death. In addition to selectivity being compromised, significantly hydrophobic AMPs (e.g. high content of leucine) have been recognised to selfassociate further reducing their ability to interact with cancer cells [31].

\section{Membrane fluidity}

AMPs can further distinguish between cancer cells and normal epithelial cells as the membrane fluidity of cancer cells is much greater [32]. The fluidity of the membrane is a key indicator in cancer progression. As cancer progresses the cell becomes more open to conformational change as the fluidity in the membrane increases [33]. AMPs act by creating pores in the membrane to gain intracellular access. As the membrane fluidity increases the AMPs can penetrate deeper into the hydrophobic layer of the cancer cells enhancing their effect [34].

\section{Cholesterol and AMPs}

AMPs have a greater impact on cancer cells because cancer cells have relatively low cholesterol content compared to normal cells $[35,36]$. This is due to the cholesterol molecules acting as membrane protectors through the modulation of membrane fluidity [37]. The cholesterol hinders the AMPs interaction with the cell membrane, reducing disruption to the target cell, restricting the chances of cell death [15].

\section{Structures of AMPs}

AMPs can be divided into four categories: (i) unstructured peptides which upon contact with membranes will fold into amphipathic $\alpha$ helical peptides (ii) $\beta$-sheet peptides stabilised by disulphide bridges (iii) $\alpha \beta$ peptides, which contain a predominance of one or two amino acids which contain disulphide bonds and (iv) non- $\alpha \beta$ that contain neither $\alpha$ helices nor $\beta$-sheets (Figure 2) [10]. The $\alpha$-helical structure plays a key role in determining the hydrophobicity of peptides. This hydrophobicity is essential for peptide secondary structure and influences the interaction of the AMP with its target cell membrane. The peptides are able to associate with each other, and in doing so, cause transmembrane pores leading to the death of the cell [25]. $\beta$ sheet peptides, which naturally originate from animals, act best as antibiotics against bacteria [38]. The $\beta$-sheet is considered the most stable of the four categories due to the hydrophobic interactions between the side chains of the peptides as well as the disulphide bridges between the chains which increases their stability [39]. $\beta$-sheet peptides are also believed to exert their antimicrobial effects through membrane disruption [40].

The $\alpha \beta$-structures contain disulphide bonds which are believed to be responsible for the structure observed in aqueous solution [41]. However, non- $\alpha \beta$ AMPs have yet to be investigated thoroughly and therefore their characteristics are not yet fully understood. So far, $\alpha-$ helical and $\beta$-sheets are the most common configurations of AMPs with anticancer properties $[14,20]$. Thus, the structure of the peptide influences the properties expressed by the AMP and can affect their potential use in cancer therapy [42].

The specific amino acid sequence of the peptide is also an important factor contributing to the properties of AMPs and their potential for anti-cancer effects. The amino acids alanine, leucine isoleucine and valine are strongly hydrophobic and are found at high levels in AMPs that exhibit anticancer properties [43]. However, altering the amino acid sequence via substitution of specific residues affects the hydrophobicity and secondary structure of the peptides [25]. For example, leucine is a large hydrophobic residue which helps to stabilise the $\alpha$-helical structure. When it is inserted into a peptide sequence the hydrophobicity of the AMP is increased along with an increase in the stability of the peptide. Yet, when leucine residues are converted to alanine residues the overall hydrophobicity of the peptide decreases. Although alanine is also hydrophobic it is small and so its short sidechain arms cannot reach the side chains of the neighbouring amino acids; this reduces the hydrophobicity of the peptide, which in turn alters its potency and thus, its therapeutic index [25].

AMPs can also be found that are rich in glycine (glycine making up $14-22 \%$ of all the amino acid residues). Glycine affects the tertiary structure formed and thus their mode of action. Glycine is a simple structural amino acid in that it does not possess a side chain, which increases the conformational flexibility of the AMP [44]. In an investigation carried out by Phoenix et al. [44] they found that glycine rich antimicrobial peptides were twice as likely to be unsuccessful in displaying anti-cancer properties. They attributed this finding to the fact that glycine rich AMPs easily undergo conformational change due to glycine's lack of a side chain. Glycine rich AMPs therefore have a reduced natural tendency to form $a$-helical structures preventing membrane binding [45]. This would suggest that the amino acid sequence of the AMP plays a pivotal role in their effectiveness as a cancer therapy.

\section{AMPs and lung cancer}

Mucins are expressed in epithelial cells within the body e.g. lung and eyes, to protect against infection. It has been recognised that in lung cancer patients, mucins are over expressed in the lung epithelial cells-specifically MUC1 and MUC2 [46]. The presence of mucins on lung cancer cells have been recognised as a negative prognosis indicator for disease-free survival [47]. However, mucins are also susceptible to glycosylation. Glycosylation is a common posttranslational modification of proteins which promotes protein folding, stability, and cell-cell interaction [48]. Mucins are characterised by repeated stretches called variable number tandem repeats (VNTRs) that are rich in serine and threonine [49]. A major type of glycosylation is the O-linked glycosylation [14]. O-glycosylation occurs when a sugar is attached to the hydroxyl group of a serine or 
threonine. As mucins are rich in these amino acids, O-glycosylation can be extensive which in turn increases the negative charge of the cancer cell membrane. In addition, the presence of the glycoprotein Alpha-1-acid glycoprotein 1, whose overexpression is significantly associated with NSCLC membranes is also said to aid in the selectivity of AMPs [50] to cancer cells. Glycosylated mucins could be advantageous from an AMP treatment perspective, by increasing the electrostatic attraction between the AMP and the lung cancer cell membrane. Furthermore, as previously mentioned, the lower cholesterol content of cancer cell membranes aids the ability of AMPs to disrupt the lipid bilayer. A study carried out by Kucharska-Newton et al. [51] showed that high density lipoprotein cholesterol and lung cancer are inversely associated meaning lung cancer cells have typically low cholesterol concentrations. Thus, the extensive glycosylation and low cholesterol content exhibited by lung cancer cell membranes suggests that AMPs could be successful anti-cancer agents to target lung cancer.

Several in vitro studies have been carried out investigating the effect of AMPs on lung cancer cell lines. In a preliminary study performed by Ohsaki et al. [52] 6 human SCLC cell lines and 4 normal human fibroblast cell lines were tested for their sensitivity to magainin A and magainin G-synthetic analogues of the frog antimicrobial peptide magainin. Results of this investigation showed that magainin $A$ and magainin $\mathrm{G}$ had anti-tumour activity to all 6 lung cancer cell lines but were not as toxic to the normal human fibroblast cell lines (against the lung cancer cell lines the average IC50 of magainin A and magainin G were 8.64 and $8.82 \mu \mathrm{M}$ respectively, while against normal human fibroblasts the IC50 of magainin A and magainin G were 21.1 and 29.2 $\mu \mathrm{M}$ respectively). Furthermore, magainin $A$ and magainin $G$ enhanced the cytotoxicity of the chemotherapeutic agents, cisplatin and etoposide when used in combination.

Huang et al. [53], carried out investigations on a custom synthesised peptide, $\mathrm{CB} 1$, derived from the natural antimicrobial peptide Cecropin B. The authors tested CB1a on normal lung epithelial cells (WI-38, MRC-5, HEL-299 cell lines), NSCLC cells (A549, NCI-H209, NCI$\mathrm{H} 460$, NCI-H520) SCLC cells (NCI-H146) and an in vivo mouse model of lung cancer The in vitro testing showed that the CB1a peptide could selectively kill lung cancer cells without affecting normal lung cells. The IC50 range for lung cancer cells was 4 to $29 \mu \mathrm{M}$ whereas for normal lung cancer cells the IC50 was significantly higher, ranging from 158 to $>300 \mu \mathrm{M}$. CB1a also significantly inhibited the growth of tumours in the in vivo mouse model. Furthermore, the authors also observed that the CB1a peptide had greater cytotoxicity to lung cancer cells (in vivo) than the chemotherapeutic agent docetaxel, while still displaying less toxicity to normal cells.

\section{AMPs in clinical trials}

To the best of our knowledge, no AMPs have entered clinical trials or the drug market to assess their efficacy as a cancer therapeutic. However peptides, as a whole, are being recognised as selective, efficacious and well tolerated therapeutic agents. As a result, in 2015 there were approximately 140 peptides drugs in clinical trials and over 500 in preclinical development [54]. Furthermore, of these peptidederived therapies several have entered clinical trials aimed at treating various types of cancers. Once such peptide is asparagine-glycinearginine tumor homing peptide (NGR-hTNF). In combination with Cisplatin, this combination therapy has undergone a phase 1 clinical trial for use in several refractory solid tumors (such as NSCLC tumors, colorectal, melanomas, sarcoma, gastric, carcinoid, malignant pleural mesothelioma (MPM) and solitary fibrous tumor of the pleura (SFTP) [55]. The patient cohort consisted of 22 patients, age range between 47 and 75 , and had previously received systemic therapy before being recruited to the trial. The solid tumour types used in the trail covered; the drug combination was delivered once every three weeks for a minimum of 6 cycles, dependent on disease progression, patient consent or increased toxicity. Concentrations of the drug combinations administered varied on an inter-patient basis. The follow up of the trial showed that seven out of the seventeen patients that completed the trial showed their disease to stabilise for a median time of 5.9 months post treatment meaning the tumor did not grow in size or metastasize during this time. One out of the seventeen patients exhibited a partial response to the treatment for 6.6 months. The most effective concentrations which showed to stabilise the disease or initiate a partial response of halting the tumor growth was found to be $0.8 \mu \mathrm{g} / \mathrm{m}^{2}$ of NGR-hTNF and $80 \mathrm{mg} / \mathrm{m}^{2}$ cisplatin. The outcome of Gregorcs' et al. [55] study was for the peptide and cisplatin combination to enter phase 2 clinical trials showing its potential as an anti-tumour agent.

Deplanque et al. [56] reported on a phase 2 clinical trial testing the peptide IM862, a natural dipeptide (L-glu-L-trp) for treatment in metastatic renal cell carcinoma (RCC). Twenty five patients were enrolled on to the trial which had histologically and/or cytologically proven metastatic RCC [56]. The treatment group was administered 20 mg of IM826 intranasally three times daily for 8 weeks. Eight of the 25 patients' RCC were reported to have stabilized-for a median of 6 months-meaning the RCC did not grow in size or metastasize to a condition worse than that prior to the trial. It is important to note the patients who saw their disease stabilize were of slow progressive RCC. Even though the drug didn't prove significant in response towards RCC, it was observed that IM826 decreased the levels of VEGF in the patients and therefore has been recommended for further investigation for its use as an anti-angiogenic agent [56].

In addition to the peptide- derived therapies undergoing clinical trials, several peptide-derived therapies are currently approved for delivery by the Food and drug administration (FDA) in the treatment of specific cancers [54]. For example Lupron TM from Abbott laboratories is on the market for use in prostate cancer and breast cancer therapy [57]. In 2011 Lupron TM alone created US $\$ 2.3$ billion in global sales- which demonstrates how peptide-derived therapies not only hold potential for anti-cancer properties, but also how lucrative the peptide-derived therapies could be to the pharmaceutical industry [54]. Thus the success of these peptides in the clinic may open the door for AMPs that have anti-cancer properties to reach the clinical settings.

However, for AMPs to be effective in vivo for lung cancer one obstacle, in particular, needs to be overcome; this is the salt concentration in the lung. Salt concentration influences the effectiveness of AMPs to function on cells. Their efficacy is believed to be greatly reduced as high salt concentrations inhibit antimicrobial activities [58]. Encouragingly, recent investigations have been carried out to improve the resistance of AMPs against increased salt concentrations. Chu et al. [59] developed a method to enhance the antimicrobial activity and salt tolerance of short antimicrobial peptides by modifying their lipophilicity with the addition of the amino acid $\beta$ naphthylanine ( $\mathrm{Nal}$ ) to their $\mathrm{N}$ or $\mathrm{C}$ terminus, In their paper, $\mathrm{Chu}$ et al. [59] designed a series of Nal-embedded peptides and tested their salttolerance (against antibiotic resistant gram negative and positive bacteria) and anticancer properties (against lung cancer cell lines PC9 and A549). Their results showed that the addition of Nal, to either the $\mathrm{N}$-terminus or embedded in the sequence of the peptide, helped the 
peptide to kill antibiotic resistant bacteria at high salt concentrations. Furthermore the Nal modified peptide displayed better anti-cancer activities than their parent peptides with the authors hypothesising that the Nal residue may help the peptide penetrate further into the cancer cell membrane, which in turn makes them more efficient at disrupting the membrane. In addition, some of the Nal modified peptides were less toxic to human red blood cells and human fibroblasts and significantly inhibited human lung tumour growth in nude mice. This study provides evidence that peptides can be designed to overcome the salt concentration restraints making AMPs a possibility for lung cancer treatment.

Furthermore the source of the AMP also plays a large role in the characteristics. For example marine animals have adapted to a very harsh environment rife with bacteria and therefore marine animals possess a diverse range of AMPs as a first line of defence [60]. AMPs from marine animals have evolved to overcome the high salinity of their natural environment giving them greater resistance to high salt concentrations than AMPs from other natural environments [60,61]. The marine AMPs resistance to high salt concentrations could be due to the substitution of lysine amino acids for arginine [60]. These properties found in marine AMPS could show further potential for their use in lung cancer therapy.

\section{Conclusion}

Survival rates for lung cancer have not shown great improvements. Many patients present with an advanced form of the disease and at this stage, the only form of treatment is chemotherapy. Not only does chemotherapy provide little success the side effects are deleterious due to the non-specific targeting of the drug. In addition, many cancers, including lung cancer develop drug resistance over time. Investigation into AMPs as anti-cancer agents is rapidly increasing. AMPs selectively target cancer cells and are not affected by the common mechanisms of chemotherapy resistance that cancer cells display. Several studies have looked into the anticancer effects of AMPs on lung cancer cell lines with promising results. Furthermore, research has shown that extensive glycosylation and low cholesterol levels, characteristic of lung cancer cell membranes, enhance the affinity of AMPs to bind to cancer cell membranes. While further investigation into the anti-cancer potential of AMPs is needed, it is possible that AMPs may offer a potentially new application in lung cancer therapy.

\section{References}

1. World Health Organisation(WHO) (2014). World Cancer Report 2014. International Agency for Research on Cancer 3.

2. Cancer Research UK (2014) Lung cancer survival statistics. Cancer research UK, London.

3. American Cancer Society (2016) Lung cancer (small cell). American Cancer Society.

4. Mackinnon A, Kopatz J, Sethi T (2010) The molecular and cellular biology of lung cancer: Identifying novel therapeutic strategies. Br Med Bull 95: 47-61.

5. Persidis A (1999) Cancer multidrug resistance. Nat Biotechnol 17: 94-95.

6. Cole SP, Bhardwaj G, Gerlach JH, Mackie JE, Grant CE, et al. (1992) Overexpression of a transporter gene in a multidrug-resistant human lung cancer cell line. Science 258: 1650-1654.

7. Huang H, Liu J, Meng Q, Niu G (2015) Multidrug resistance protein and topoisomerase 2 alpha expression in non-small cell lung cancer are related with brain metastasis postoperatively. Int J Clin 8: 11537-11542.

8. Pinheiro da Silva F, Machado MCC (2012) Antimicrobial peptides: clinical relevance and therapeutic implications. Peptides 36: 308-314.
9. Tian Y, Wang H, Li B, Ke M, Wang J, et al. (2013) The cathelicidin-BF Lys16 mutant Cbf-K16 selectively inhibits non-small cell lung cancer proliferation in vitro. Oncol Rep 30: 2502-2510.

10. Hancock RE, Diamond G (2000) The role of cationic antimicrobial peptides in innate host defences. Trends Microbiol 8: 402-410.

11. Izadpanah A, Gallo RL (2005) Antimicrobial peptides. J Am Acad Dermatol 52: 381-392.

12. Guaní-Guerra E, Santos-Mendoza T, Lugo-Reyes SO, Terán LM (2010) Antimicrobial peptides: general overview and clinical implications in human health and disease. Clin Immunol 135: 1-11.

13. Zhao X, Wu H, Lu H, Li G, Huang Q (2013) LAMP: A Database Linking Antimicrobial Peptides. PLoS One 8: e66557.

14. Hoskin DW, Ramamoorthy A (2008) Studies on anticancer activities of antimicrobial peptides. Biochim Biophys Acta 1778: 357-375.

15. Gaspar D, Veiga AS, Castanho MA (2013) From antimicrobial to anticancer peptides. A review. Front Microbiol 4: 294.

16. Lee J, Lee DG (2015) Antimicrobial Peptides (AMPs) with dual mechanisms: Membrane disruption and apoptosis. I Microbiol Biotechnol 25: 759-764.

17. Chang WK, Wimley WC, Searson PC, Hristova K, Merzlyakov M (2009) Characterization of antimicrobial peptide activity by electrochemical impedance spectroscopy. Biochim Biophys Acta 1778: 2430-2436.

18. Park Y, Hahm KS (2005) Antimicrobial peptides (AMPs): peptide structure and mode of action. J Biochem Mol Biol 38: 507-516.

19. Mihajlovic M, Lazaridis T (2010) Antimicrobial peptides in toroidal and cylindrical pores. Biochim Biophys Acta 1798: 1485-1493.

20. Liu Y, Li Y, Li Z, Lan X, Leung PHM, et al. (2015) Mechanism of anticancer effects of antimicrobial peptides. Journal of Fiber Bioengineering and Informatics. 8: 25-36.

21. Su Y, Waring AJ, Ruchala P, Hong M (2011) Structures of $\beta$-hairpin antimicrobial protegrin peptides in lipopolysaccharide membranes: mechanism of gram selectivity obtained from solid-state nuclear magnetic resonance. Biochemistry 50: 2072-2083.

22. Bahar AA, Ren D (2013) Antimicrobial Peptides. Pharmaceuticals. 6: 1543-1575.

23. Magalhaes A, Maigret B, Hoflack J, Gomes JN, Scheraga HA (1994) Contribution of unusual arginine-arginine short range interactions to stabilization and recognition of proteins. J Protein Chem 13: 195-215.

24. He Y, Lazaridis T (2013) Activity determinants of helical antimicrobial peptides: a large-scale computational study. PLoS One 8: e66440.

25. Huang YB, Wang XF, Wang HY, Liu Y, Chen Y (2011) Studies on mechanism of action of anticancer peptides by modulation of hydrophobicity within a defined structural framework. Mol Cancer Ther 10: 416-426.

26. Wang G (2014) Human antimicrobial peptides and proteins. Pharmaceuticals 7: 545-594.

27. Hancock RE, Chapple DS (1999) Peptide antibiotics. Antimicrob Agents Chemother 43: 1317-1323.

28. Utsugi T, Schroit AJ, Connor J, Bucana CD, Fidler IJ (1991) Elevated expression of phosphatidylserine in the outer membrane leaflet of human tumor cells and recognition by activated human blood monocytes. Cancer Res 51: 3062-3066.

29. Lee JK, Gopal R, Park SC, Ko HS, Kim Y, et al. (2013) A proline-hinge alters the characteristics of the amphipathic $\alpha$-helical AMPs. PLoS One 8: e67597.

30. Henriksen JR, Etzerodt T, Gjetting T, Andresen TL (2014) Side chain hydrophobicity modulates therapeutic activity and membrane selectivity of antimicrobial peptide mastoparan-X. PLoS One 9: e91007.

31. Findlay B, Zhanel GG, Schweizer F (2010) Cationic amphiphiles, a new generation of antimicrobials inspired by the natural antimicrobial peptide scaffold. Antimicrob Agents Chemother. 54: 4049-4058.

32. Kozłowska K, Witkowski JM, Zarzeczna M, Cichorek M (1999) Diversity of the plasma membrane properties of transplantable hamster melanomas with regard to the expression of P-glycoprotein. Folia Histochem Cytobiol 37: 173-177. 
Page 6 of 6

33. Coughlin MF, Bielenberg DR, Lenormand G, Marinkovic M, Waghorne CG, et al. Cytoskeletal stiffness, friction, and fluidity of cancer cell lines with different metastatic potential. Clin Exp Metastasis 30: 237-250.

34. Mehla J, Sood SK (2013) Connecting membrane fluidity and surface charge to pore-forming antimicrobial peptides resistance by an ANNbased predictive model. Appl Microbiol Biotechnol 97: 4377-4384.

35. Simons K, Ikonen E (2000) How cells handle cholesterol. Science 290: 1721-1726.

36. Leuschner C, Hansel W (2004) Membrane disrupting lytic peptides for cancer treatments. Curr Pharm Des 10: 2299-2310.

37. Schweizer F (2009) Cationic amphiphilic peptides with cancer-selective toxicity. Eur J Pharmacol 625: 190-194

38. Panteleev PV, Bolosov IA, Balandin SV, Ovchinnikova TV (2015) Structure and biological functions of $\beta$-hairpin antimicrobial peptides. Acta Naturae 7: 37-47.

39. Wang J, Mura M, Zhou Y, Pinna M, Zvelindovsky AV, et al. (2014) The cooperative behaviour of antimicrobial peptides in model membranes. Biochim Biophys Acta 1838: 2870-2881.

40. Jin Y, Hammer J, Pate M, Zhang Y, Zhu F, et al. (2005) Antimicrobial activities and structures of two linear cationic peptide families with various amphipathic beta-sheet and alpha-helical potentials. Antimicrob Agents Chemother 49: 4957-4964.

41. Wang G (2013) Database-guided discovery of potent peptides to combat HIV-1 or superbugs. Pharmaceuticals 6: 728-758.

42. Lin MC, Hui CF, Chen JY, Wu JL (2013) Truncated antimicrobial peptides from marine organisms retain anticancer activity and antibacterial activity against multidrug-resistant Staphylococcus aureus. Peptides 44: 139-148.

43. Wang G, Li X, Wang Z (2009) APD2: the updated antimicrobial peptide database and its application in peptide design. Nucleic Acids Res 37: 933-937.

44. Phoenix D, Dennison SR, Harris F (2013) Antimicrobial peptides. Weinheim: Wiley-VCH.

45. Harris F, Dennison SR, Singh J, Phoenix DA (2013) On the selectivity and efficacy of defense peptides with respect to cancer cells. Medicinal Research Reviews 33: 190-234.

46. Gao WM, Kuick R, Orchekowski RP, Misek DE, Qiu J, et al. (2005) Distinctive serum protein profiles involving abundant proteins in lung cancer patients based upon antibody microarray analysis. BMC Cancer 5 : 110.

47. Graziano SL, Lacas B, Vollmer R, Kratzke R, Popper H, et al. (2013) Cross- validation analysis of the prognostic significance of mucin expression in patients with resected non-small cell lung cancer treated with adjuvant chemotherapy: results from IALT, JBR.10 and ANITA. Lung Cancer 82: 149-155.

48. Marien E, Meister M, Muley T, Fieuws S, Bordel S, et al (2015) Non-small cell lung cancer is characterized by dramatic changes in phospholipid profiles. Int J Cancer. 137: 1539-1548.

49. Varki A (2009) Essentials of glycobiology (2nd edn) Cold Spring Harbor, N.Y, Cold Spring Harbor Laboratory Press.

50. Ayyub A, Saleem M, Fatima I, Tariq A, Hashmi N, et al. (2015) Glycosylated Alpha-1-acid glycoprotein 1 as a potential lung cancer serum biomarker. Int J Biochem Cell Biol 70: 68-75.

51. Kucharska-Newton AM, Rosamond WD, Schroeder JC, McNeill AM, Coresh J, et al. (2008) HDL-cholesterol and the incidence of lung cancer in the Atherosclerosis Risk in Communities (ARIC) study. Lung Cancer 61: 292-300.

52. Ohsaki Y, Gazdar AF, Chen HC, Johnson BE (1992) Antitumor activity of magainin analogues against human lung cancer cell lines. Cancer Research 52: 3534-3538.

53. Huang CY, Huang HY, Forrest MD, Pan YR, Wu WJ, et al. (2014) Inhibition effect of a custom peptide on lung tumors. PLoS One 9: e109174.

54. Fosgerau K, Hoffmann T (2015) Peptide therapeutics: current status and future directions. Drug Discov Today 20: 122-128.

55. Gregorc V, De Braud FG, De Pas TM, Scalamogna R, Citterio G, et al. (2011) Phase I study of NGR-hTNF, a selective vascular targeting agent, in combination with cisplatin in refractory solid tumors. Clin Cancer Res 17: 1964-1972.

56. Deplanque G, Madhusudan S, Jones PH, Wellmann S, Christodoulos K, et al. (2004) Phase II trial of the antiangiogenic agent IM862 in metastatic renal cell carcinoma. Br J Cancer 91: 1645-1650.

57. Kaspar AA, Reichert JM (2013) Future directions for peptide therapeutics development. Drug Discov Today 18: 807-817.

58. Bals R, Goldman MJ, Wilson JM (1998) Mouse B-Defensin 1 is a saltsensitive Antimicrobial peptide present in epithelia of the lung and urogenital tract. Infection and Immunity 66: 1225-1232.

59. Chu HL, Yu HY, Yip BS, Chih YH, Liang CW, et al. (2013) Boosting salt resistance of short antimicrobial peptides. Antimicrob Agents Chemother 57: 4050-4052.

60. Falanga A, Lombardi L, Franci G, Vitiello M, Iovene MR, et al. (2016) Marine antimicrobial peptides: nature provides templates for the design of novel compounds against pathogenic bacteria. Int J Mol Sci 17.

61. Patrzykat A, Douglas SE (2003) Gone gene fishing: how to catch novel marine antimicrobials. Trends Biotechnol 21:362-369. 